\title{
STABILITY OF BARRELLEDNESS AND RELATED CONCEPTS IN TOPOLOGICAL VECTOR SPACES
}

\author{
(Received 9th March 1984)
}

\author{
by J. O. POPOOLA and I. TWEDDLE
}

\section{Introduction}

Let $E$ be a separated locally convex barrelled space with continuous dual $E^{\prime}$ and algebraic dual $E^{*}$ and let $M$ be a subspace of $E^{*}$ with $M \cap E^{\prime}=\{0\}$ and $\operatorname{dim} M \leqq \aleph_{0}$. Robertson, Tweddle and Yeomans have recently considered the question of barrelledness under the Mackey topology $\tau\left(E, E^{\prime}+M\right)$ when $E$ is given to be barrelled under its original topology $\tau\left(E, E^{\prime}\right)[5],[6],[7]$.

Perhaps the simplest way of refining the topology of any topological vector space on which there are discontinuous linear functionals is by adding enough "weak neighbourhoods" to make some such functionals continuous. It therefore seems reasonable to consider related questions and procedures in topological vector spaces which are not necessarily locally convex or separated. We are able to extend the main stability results of [5] and [7] to barrelled topological vector spaces and we have some partial results on the preservation of ultrabarrelledness and hyperbarrelledness, which are rather more important concepts in the non-locally convex cases.

The first author gratefully acknowledges the award of a Senior Fellowship by the British Council.

\section{Preliminaries}

Let $E(\xi)$ be a topological vector space and let $M$ be a subspace of its algebraic dual $E^{*}$. If $\mathscr{U}$ is a base of neighbourhoods of the origin for $\xi$, then the sets

$$
U \cap\left\{x:\left|\left\langle x, x_{i}^{\prime}\right\rangle\right| \leqq 1, i=1,2, \ldots, n\right\}
$$

where $U \in \mathscr{U}, n \in \mathbb{N}$ and the $x_{i}^{\prime} \in M$, form a base of neighbourhoods of the origin for a vector topology $\xi[M]$ on $E$. In fact $\xi[M]$ is the coarsest vector topology on $E$ which is finer than $\xi$ and which makes the elements of $M$ continuous. We first characterise the $\xi[M]$-continuous linear functionals.

Lemma 1. A linear functional on $E$ is $\xi[M]$-continuous if and only if it is of the form $g+h$ where $g$ is an $\xi$-continuous linear functional and $h \in M$.

Proof. Suppose that $f$ is an $\xi[M]$-continuous linear functional. Then there are $U \in \mathscr{U}$ and $x_{1}^{\prime}, x_{2}^{\prime}, \ldots, x_{n}^{\prime} \in M$ such that $f$ is bounded on

$$
W=U \cap\left\{x:\left|\left\langle x, x_{i}^{\prime}\right\rangle\right| \leqq 1, \quad i=1,2, \ldots, n\right\} .
$$


Let $F=\left\{x \in E:\left\langle x, x_{i}^{\prime}\right\rangle=0, i=1,2, \ldots, n\right\}$ and let $G$ be an algebraic supplement of $F$ in $E$. Note that $G$ is finite dimensional.

Since $W \cap F=U \cap F$ it follows that $\left.f\right|_{F}$ is $\left.\xi\right|_{F}$-continuous. We can extend $\left.f\right|_{F}$ by continuity to a linear functional $f_{1}$ on the $\xi$-closure $F_{1}$ of $F$. Then, since $E(\xi)$ is the topological direct sum of $F_{1}\left(\left.\xi\right|_{F_{1}}\right)$ and a finite dimensional supplement, any linear extension $g$ of $f_{1}$ to the whole of $E$ is $\xi$-continuous. Choose such a $g$ and put $h=f-g$. We have $\left.h\right|_{F}=0$, which implies that $h \in \operatorname{span}\left\{x_{1}^{\prime}, x_{2}^{\prime}, \ldots, x_{n}^{\prime}\right\}$ since otherwise we would be able to find $a \in F$ with $\langle a, h\rangle \neq 0$. Thus $f=g+h$ where $g$ is $\xi$-continuous and $h \in \operatorname{span}\left\{x_{1}^{\prime}, x_{2}^{\prime}, \ldots, x_{n}^{\prime}\right\} \subseteq M$. Conversely, it is clear that any linear functional of this form is $\xi[M]$-continuous.

Lemma 2. Let $M=\bigcup_{k=1}^{\infty} M_{k}$ where for each $k, M_{k} \subseteq M_{k+1}$ and $M_{k}$ is a finite dimensional subspace of $E^{*}$. For any subset $X$ of $E$, let $X_{0}$ be its $\xi[M]$-closure and $X_{k}$ its $\xi\left[M_{k}\right]$-closure. Then $X_{0}=\bigcap_{k=1}^{\infty} X_{k}$.

Proof. Clearly $X_{0} \subseteq X_{k} \forall k \in \mathbb{N}$ so that $X_{0} \subseteq \bigcap_{k=1}^{\infty} X_{k}$. On the other hand if $z \notin X_{0}$, we can find $U \in \mathscr{U}$ and $x_{1}^{\prime}, x_{2}^{\prime}, \ldots, x_{n}^{\prime} \in M$ for some $n \in \mathbb{N}$ such that

$$
X \cap\left(z+U \cap\left\{x:\left|\left\langle x, x_{i}^{\prime}\right\rangle\right| \leqq 1, i=1,2, \ldots, n\right\}\right)=\emptyset .
$$

Now there must exist $k_{0} \in \mathbb{N}$ such that $x_{i}^{\prime} \in M_{k_{0}}(i=1,2, \ldots, n)$, which implies that $U \cap\left\{x:\left|\left\langle x, x_{i}^{\prime}\right\rangle\right| \leqq 1, i=1,2, \ldots, n\right\}$ is a neighbourhood of the origin for $\xi\left[M_{k_{0}}\right]$. Thus $z \notin X_{k_{0}}$, which shows that $\bigcap_{k=1}^{\infty} X_{k} \subseteq X_{0}$.

If $M$ is finite dimensional we shall say that $\xi[M]$ is a finite extension of $\xi$. We shall use $E^{\prime}$ to denote the space of $\xi$-continuous linear functionals. If $E^{\prime}$ has countably infinite codimension in $E^{\prime}+M$ we shall say that $\xi[M]$ is a countable extension of $\xi$. The term countable will always mean countably infinite.

In a non-locally convex topological vector space the bipolar property may fail, i.e. for a closed absolutely convex set $A$ we may not have $A=A^{\circ \circ}$. A closed absolutely convex neighbourhood of the origin always satisfies the bipolar property. We will also require the following elementary result in this connection.

Lemma 3. Let $B=\cap\left\{B_{\lambda}: \lambda \in \Lambda\right\}$, where each $B_{\lambda}$ is a closed absolutely convex set which satisfies the bipolar property in a topological vector space $E(\xi)$. Then $B$ satisfies the bipolar property.

Proof. Taking polars in $E^{\prime}$ we have $B^{\circ} \supseteq\left\{B_{\lambda}^{\circ}: \lambda \in \Lambda\right\}$ and so $B \subseteq B^{\circ \circ} \subseteq \cap\left\{B_{\lambda}^{\circ \circ}: \lambda \in \Lambda\right\}$ $=\cap\left\{B_{\lambda}: \lambda \in \Lambda\right\}=B$.

We shall use $c$ to denote the cardinal number of $\mathbb{R}$. A subset of a vector space is said to be $c$-dimensional if its linear span has dimension $c$.

\section{Some stability results}

We begin by recalling some definitions (see [1], [2], [4], [8] for further details). The 
first four refer to subsets of a topological vector space $E(\xi)$ which need not be separated or locally convex.

(i) A barrel is a closed absolutely convex absorbent set.

(ii) A set $A$ is semiconvex if there is $\lambda>0$ such that $A+A \subseteq \lambda A$.

(iii) A hyperbarrel is a closed balanced absorbent semiconvex set.

(iv) An ultrabarrel is a sequence $\left(U_{n}\right)$ of closed balanced absorbent sets such that $U_{n+1}+U_{n+1} \subseteq U_{n}$ for each $n \in \mathbb{N}$.

(v) $E(\xi)$ is barrelled, resp. hyperbarrelled if each barrel, resp. hyperbarrel is an $\xi$ neighbourhood of the origin; it is ultrabarrelled if the elements of each ultrabarrel are $\xi$-neighbourhoods of the origin. It is well-known and easy to see that ultrabarrelled $\Rightarrow$ hyperbarrelled $\Rightarrow$ barrelled.

For simplicity we state Theorems 1 and 2 for ultrabarrelled spaces but it will be clear that they hold also for hyperbarrelled or barrelled spaces.

Theorem 1. Let $\xi[M]$ be any finite extension of $\xi$. If $E(\xi)$ is ultrabarrelled then so also is $E(\xi[M])$.

Proof. Let $F=\left\{x:\left\langle x, x^{\prime}\right\rangle=0 \forall x^{\prime} \in M\right\}$. Then $F$ is an $\xi[M]$-closed, finite codimensional subspace of $E$. Further $\xi$ and $\xi[M]$ induce the same topology on $F$ and $E(\xi[M])$ is the topological direct sum of $F\left(\left.\xi\right|_{F}\right)$ and any supplement. The result now follows since ultrabarrelledness is inherited by finite codimensional vector subspaces [1, Proposition 3.1].

We now consider countable extensions. For the locally convex case it is shown in [5, Theorem 3] that if $E^{\prime} \neq E^{*}$ it is always possible to find a countable extension which does not preserve barrelledness (see also [6, Theorem 2]). Since an ultrabarrelled space is barrelled in its associated locally convex topology [4, Proposition 19], we may deduce a corresponding result for ultrabarrelled spaces and likewise for hyperbarrelled spaces. In the positive direction an application of Theorem 1 allows us to identify a simple class of spaces for which countable extensions, which preserve the given property, do exist.

Theorem 2. Let $E(\xi)$ be the topological product of the ultrabarrelled spaces $E_{n}\left(\xi_{n}\right)$ $(n \in \mathbb{N})$ and suppose that for each $n \in \mathbb{N}, E_{n}^{\prime} \neq E_{n}^{*}$. (*) Then there is a countable extension $\xi[M]$ under which $E$ is ultrabarrelled.

Proof. For each $m \in \mathbb{N}$ choose $f_{m} \in E_{m}^{*} \backslash E_{m}^{\prime}$ and define $g_{m}$ on $E$ by $g_{m}\left(\left(x_{n}\right)\right)=f_{m}\left(x_{m}\right)$. Let $L_{n}$ be the span of $f_{n}$ in $E_{n}^{*}$ and let $M=\operatorname{span}\left\{g_{m}: m \in \mathbb{N}\right\}$. Then it is easy to see that

(i) $M \cap E^{\prime}=\{0\}$,

(ii) $M$ has countable dimension,

(iii) $\xi[M]$ is the product topology defined by the topologies $\xi_{n}\left[L_{n}\right]$.

The result follows since by Theorem $1, E_{n}\left(\xi_{n}\left[L_{n}\right]\right)$ is ultrabarrelled and any product of ultrabarrelled space is ultrabarrelled. 
Remark. Theorem 2 may be extended to any infinite product of ultrabarrelled spaces as long as $\left(^{*}\right)$ applies to some countable subset of the factors.

In the case of barrelledness we are able to remove the assumptions of separatedness and local convexity in Theorem 5 of [5] and Theorem 1 of [7]. The duality arguments of [5] and [7] are reformulated in terms of the results of Section 2.

Theorem 3. Let $E(\xi)$ be a barrelled space with a dense c-codimensional barrelled subspace. Then there is a countable extension $\xi[M]$ under which $E$ is barrelled.

Proof. Let $L$ be a dense $c$-codimensional barrelled subspace of $E$ and let $G$ be an algebraic supplement of $L$ in $E$. Exactly as in [5] we identify $G$ algebraically with $\omega\left(=\mathbb{R}^{N}\right.$ or $\left.\mathbb{C}^{N}\right)$ and extend each element of the dual $\phi\left(=\mathbb{R}^{(N)}\right.$ or $\left.\mathbb{C}^{(N)}\right)$ of $\omega$ to a linear functional on $E$ by putting it equal to zero on $L$. The set $M$ of all these extensions is a countable dimensional subspace of $E^{*}$ with $E^{\prime} \cap M=\{0\}$. Let $e_{1}, e_{2}, e_{3}, \ldots$ be a basis in $M$ and for each $k \in \mathbb{N}$ put $M_{k}=\operatorname{span}\left\{e_{1}, e_{2}, \ldots, e_{k}\right\}$.

Let $B$ be a barrel in $E(\xi[M])$ and let $B_{k}$ be the $\xi\left[M_{k}\right]$-closure of $B(k \in \mathbb{N})$. Then by Lemma 2 we have $B=\bigcap_{k=1}^{\infty} B_{k}$. By Theorem $1 E\left(\xi\left[M_{k}\right]\right)$ is barrelled and so $B_{k}$ is a neighbourhood of the origin under $\xi\left[M_{k}\right]$ and therefore also under the finer topology $\xi[M]$. Since all the $B_{k}$ are therefore $\xi[M]$-closed absolutely convex neighbourhoods of the origin in $E(\xi[M])$, we obtain from Lemma 3 that $B$ satisfies the bipolar property. Let $C, D$ be respectively the projections of $B^{\circ}$ in $E^{\prime}$ and $M$.

Since $\xi[M]$ and $\xi$ induce the same topology on $L$ we deduce that $B \cap L$ is a closed absolutely convex neighbourhood of the origin in $L\left(\left.\xi\right|_{L}\right)$ and by denseness its closure $A$ in $E(\xi)$ is an $\xi$-neighbourhood of the origin. Then by the bipolar property and the fact that $M$ annihilates $L$, we have on taking polars in $E^{\prime}$ that $C \subseteq(B \cap L)^{\circ}=A^{\circ}$. Consequently $C$ is pointwise bounded on $E$. Since the same is true of $B^{\circ}$, it follows that $D$ is also pointwise bounded on $E$ and so from the construction of $M$, we deduce that $D$ must be contained in $M_{k}$ for some $k \in \mathbb{N}$. For this $k$ we then have $B^{\circ} \subseteq C+D \subseteq E^{\prime}+M_{k}$. It now follows that $B$ is a neighbourhood of the origin under $\xi\left[M_{k}\right]$ and therefore also under $\xi[M]$.

Theorem 4. Let $E(\xi)$ be a barrelled space in which there is a c-dimensional semiconvex bounded set. Then there is a countable extension $\xi[M]$ under which $E$ is barrelled.

Proof. Let $A$ be a semiconvex bounded subset of $E$ which spans a $c$-dimensional subspace $G$ of $E$. Since the balanced hull of $A$ is bounded, semiconvex and has the same span as $A$, we may assume that $A$ is balanced. The set of positive multiples of $A$ then forms a base of neighbourhoods of the origin for a locally bounded topology $\eta$ on $G$ which is finer than $\left.\xi\right|_{G}$. As in [7] we consider an algebraic isomorphism $t: G \rightarrow \omega$. Since $G^{\prime}$ is normed by $\left\|x^{\prime}\right\|=\sup \left\{\left|\left\langle x, x^{\prime}\right\rangle\right|: x \in A\right\}$, we deduce in the same way that $t^{\prime}(\phi) \cap G^{\prime}$ has countable codimension in $t^{\prime}(\phi)$. Let $N$ be an algebraic supplement of $t^{\prime}(\phi) \cap G^{\prime}$ in $t^{\prime}(\phi)$. We extend each element of $N$ to a linear functional on $E$ by putting it equal to zero on some fixed algebraic supplement of $G$ in $E$. The set $M$ of these extensions is then a countable dimensional subspace of $E^{*}$ with $E^{\prime} \cap M=\{0\}$.

Let $B$ be a barrel in $E(\xi[M])$. It follows that $C=B \cap G$ is a barrel in $G(\eta[N])$ and so, 
taking the polar in $G^{\prime}+N$, we have that $C^{\circ}$ is pointwise bounded on $G$. Using the same normalisation technique as in [7], we see that $C^{\circ} \subseteq G^{\prime}+Q$ where $Q$ is a finite dimensional subspace of $N$. Let $P$ be the subspace of $M$ obtained by extending the elements of $Q$ in the prescribed manner.

Suppose $f \in E^{\prime}+M$ and $f$ is bounded on $B$. Then $\left.f\right|_{G}$ is an element of $G^{\prime}+N$ which is bounded on $C$, so that $\left.f\right|_{G} \in G^{\prime}+Q$. It follows that $f \in E^{\prime}+P$ and consequently that $B^{\circ} \subseteq E^{\prime}+P$. The result now follows as in Theorem 3 since $B$ again satisfies the bipolar property.

\section{Remarks.}

(i) An infinite dimensional complete metrizable topological vector space always has $c$-dimensional bounded absolutely convex sets [8, II.5; 3, proof].

(ii) It would be of interest to know if barrelledness can be replaced by hyperbarrelledness or even ultrabarrelledness in Theorems 3 and 4.

\section{REFERENCES}

1. S. O. Iyahen, On certain classes of linear topological spaces, Proc. London Math. Soc. 18 (1968), 285-307.

2. S. O. Iyahen, Semiconvex spaces, Glasgow Math. J. 9 (1967), 111-118.

3. J. O. Popoola and I. Twedde, On the dimension of a complete metrizable topological vector space, Canad. Math. Bull. 20 (1977), 271-2.

4. W. J. Robertson, Completeness of topological vector spaces, Proc. London Math. Soc. 8 (1958), 242-257.

5. W. J. Robertson, I. Twedde and F. E. Yeomans, On the stability of barrelled topologies, III, Bull. Austral. Math. Soc. 22 (1980), 99-112.

6. W. J. Robertson and F. E. Yeomans, On the stability of barrelled topologies, I, Bull. Austral. Math. Soc. 20 (1979), 385-395.

7. I. Tweddle and F. E. Yeomans, On the stability of barrelled topologies II, Glasgow Math. J. 21 (1980), 91-95.

8. L. WAeLBroecK, Topological Vector Spaces and Algebras (Lecture Notes in Math., Vol. 230, Springer-Verlag, Berlin and New York, 1971).

UNIVERSITY OF LAGOS

LAGOS

NIGERIA

UNIVERSity OF STIRLING

STIRLING

FK9 4LA 